\title{
Kinderbetreuungsrecht und Unterhalt im Wandel: Zahlt Frau drauf?*
}

\author{
Gretel Diehl \\ Vorsitzende Richterin am Oberlandesgericht, Frankfurt
}

Die Antwort auf diese Frage ist natürlich die typische Juristen bzw. Juristinnenantwort, nämlich: Es kommt darauf an. Und wie so oft im juristischen Berufsalltag ist die Antwort auch hier richtig. Es kommt nämlich ganz entscheidend darauf an, unter welchem Gesichtspunkt man die Frage betrachtet.

Wird sie rein wirtschaftlich betrachtet, also nur gemessen an der Höhe und der Dauer des Ehegattenunterhaltes, dann heißt die Antwort eindeutig ja, jedenfalls soweit es die unschuldig geschiedenen Frauen betrifft. Denn seit der Familienrechtsreform 1977 haben der Gesetzgeber und die Rechtsprechung die unterhaltsrechtliche Situation dieser Ehefrauen und Mütter kontinuierlich verschlechtert. Dies betrifft nicht nur die Dauer und die Höhe des Unterhaltsanspruches, sondern auch die Pflicht zur Sicherung des Bedarfes durch eigene Erwerbstätigkeit. Nach den im Ehegesetz enthaltenen Unterhaltsregelungen stand der unschuldig geschiedenen Ehefrau ein lebenslanger Unterhaltsanspruch zu, wobei die Höhe durch das zur Verteilung vorhandene Geld und die Anzahl der davon leben müssenden Personen bestimmt wurde und die Verteilung oft mit Hilfe eines bestimmten Schlüssels erfolgte. Bekannt und vielfach angewandt wurden damals der sogenannte Kölner Schlüssel aber vor allem der Zwickauer Schlüssel, der teilweise heute noch im Rahmen der Berechnung der anteiligen Wohnkosten im Unterhaltsrecht Anwendung findet.

Bedingung für den lebenslangen Unterhaltsanspruch der Ehefrau im Westen war damals nur eines: Die Frau musste unschuldig geschieden worden sein. Wollte der Mann also seiner lebenslangen Unterhaltspflicht entgehen, musste ihm der Nachweis gelingen, dass die Ehefrau schuld an der Ehescheidung war. Diese rechtlichen Regelungen wurden 1977 zugunsten des neuen Ehescheidungsrechtes, das das Zerrüttungsprinzip einführte, aufgegeben und damit einhergehend wurden neue Regelungen zum Unterhalt erforderlich, auf die noch eingegangen werden soll.

Denn zunächst zurück zur ursprünglichen Fragestellung:

Wenn frau jedoch nicht nur die wirtschaftliche Seite der Fragestellung betrachtet, sondern auch die im Rahmen der Reform 1977 erfolgte verbesserte Gleichstellung der Frauen, dann fällt die Antwort keineswegs so eindeutig aus. Um dies zu illustrieren reicht ein Blick auf die rechtlichen Regelungen zur Arbeitstätigkeit von Ehefrauen außerhalb des Familienheimes bzw. des Familienbetriebes.

Die frühere DDR war in der rechtlichen Gleichstellung von Männern und Frauen dabei - jedenfalls auf dem Papier - bereits 1966 viel weiter als die frühere Bundesrepublik Deutschland. Nach dem Familiengesetz der DDR wurde ab 1966 die Verpflichtung aufgestellt, dass die Ehepartner ihre Verbindung so gestalten, dass die Frau ihre berufliche und gesellschaftliche Tätigkeit mit der Mutterschaft vereinbaren kann.

In der früheren Bundesrepublik Deutschland galt dagegen von 1958 bis zum Inkrafttreten der Familienrechtsreform am 1. Juli 1977 folgende Fassung des $\mathbb{S} 1356$ Abs. 1 BGB:

„Die Frau führt den Haushalt in eigener Verantwortung. Sie ist berechtigt, erwerbstätig zu sein, soweit dies mit ihren Pflichten in Ehe und Familie vereinbar ist. " 1 Ehefrauen benötigten daher die Zustimmung ihrer Ehemänner, wenn sie eine Erwerbstätigkeit aufnehmen wollten und mussten diese Zustimmung des Mannes dem Arbeitgeber schriftlich vorlegen, um einen Arbeitsvertrag wirksam abzuschließen.

Die gesetzlichen Neuregelungen anlässlich der Reform 1977 sind Folge des gesellschaftlichen Wandels in dieser Zeit, vor allem der Emanzipationsbewegung der 60er und 70er Jahre, aber natürlich auch der ökonomischen Veränderungen. Je stärker der Bedarf an Arbeitskräften wurde, umso größer wurde auch die Bereitschaft die Ressource „Frauenarbeitskraft“ in Anspruch zu nehmen. Das Interesse und der Bedarf der Wirtschaft an gut ausgebildeten Mädchen und Frauen nahmen zu und bedingten ein verändertes Verständnis der Rolle der Frau. Zugleich wurde der Wunsch, sich mehr Konsumgüter leisten zu können, immer stärker. Trotz all dieser Veränderungen aber blieb in den westlichen Bundesländern über lange Jahre die Vorstellung erhalten, dass Kinder am besten von ihren Müttern betreut werden und sich Kindererziehung und Erwerbstätigkeit - jedenfalls bis zu einem bestimmten Alter des Kindes - nicht miteinander vereinbaren lassen. Darauf gründet auch die Rechtsprechung, dass eine Mutter sich frei und ohne gegen ihre unterhaltsrechtlichen Verpflichtungen zu verstoßen für die Kinderbetreuung an Stelle von Arbeitstätigkeit entscheiden kann. Auf dieser wieder fußt ein juristisches Modell, dass nach seiner Erfindung viele Jahre lang die Rechtsprechung zum Unterhaltsanspruch einer Kinder betreuenden Mutter geprägt hat - das sogenannte Altersphasenmodell.

Dieses wurde herangezogen bei der Prüfung, ab welchem Alter eines Kindes der betreuenden Mutter im Rahmen ihres Ehegattenunterhaltsanspruches eine Berufstätigkeit neben der Kinderbetreuung zugemutet werden kann. Das Altersphasenmodell wurde scherzhaft auch 0-8-15 Modell genannt, denn in der Regel wurde davon ausgegangen, dass die betreuende Ehefrau bis zur Vollendung des achten Lebensjahres des jüngsten Kindes gar nicht, danach teilschichtig und erst ab Vollendung des 15. Lebensjahres Vollzeit arbeiten müsse. Natürlich waren dabei

\footnotetext{
Vortrag auf der Fachtagung „Am Ende geht's ums Geld. Auseinandersetzung und Teilhabe. Geschlechtergerechtigkeit im Familienrecht" am 28. September 2013, im Rahmen des 40. Bundeskongresses des djb in Leipzig.

1 Die heutige Fassung des § 1356 BGB lautet: „Die Ehegatten regeln die Haushaltsführung in gegenseitigem Einvernehmen [...]. Beide Ehegatten sind berechtigt, erwerbstätig zu sein."
} 
auch immer die besonderen Gegebenheiten in der Familie zu berücksichtigen, sodass individuelle Abweichungen von diesen Altersphasen möglich waren. In der täglichen Praxis bot das Altersphasenmodell sowohl den Anwälten/innen im Rahmen der Beratung der Ehefrauen als auch den Familienrichter/innen gute Anhaltspunkte für die Entscheidung der Frage, ob einer Mutter neben der Kinderbetreuung eine Arbeitstätigkeit zumutbar war und wenn ja, in welchem zeitlichen Umfang. Hinzu kam, dass der Ehegattenunterhaltsanspruch und der Kindesunterhaltsanspruch im Mangelfall den gleichen Rang hatten. Wenn das für den Unterhalt zur Verfügung stehende Einkommen des Unterhaltspflichtigen nicht ausreichte, um den Ehegatten- und den Kindesunterhaltsbedarf zu decken, wurde der verfügbare Betrag zwischen Ehefrau und minderjährigen Kindern nach einer Quote entsprechend dem jeweiligen Bedarf verteilt.

Parallel dazu bestanden auch im öffentlichen Leistungsrecht Vorgaben, die das Altersphasenmodell stützten. Dies galt zum einen für die Arbeitsverpflichtung im Rahmen der Arbeitslosenhilfe wie auch der früheren Sozialhilfe nach dem BSHG. Die Kinderbetreuung hinderte zunächst weitgehend, später mit gewissen Einschränkungen ab dem dritten Lebensjahr des Kindes die Verpflichtung, im Rahmen der öffentlichen Leistungsgewährung selbst Arbeitseinkünfte erzielen zu müssen. An den erziehenden Elternteil wurde zudem Erziehungsgeld nach dem Bundeserziehungsgeldgesetz gezahlt, das nicht als Lohnersatz galt und daher auf öffentliche Leistungen, die Lohnersatzfunktion hatten, nicht angerechnet wurde. Unterhaltsvorschussleistungen wurden (und werden) erbracht, ohne dass das Einkommen des betreuenden Elternteils eine Rolle spielt, aber es gab - anders als heute - keine breitgefächerte Diskussion, dies zu ändern, denn es war selbstverständlich, dass wirtschaftlich gut situierte Mütter keinen solchen Antrag stellten bzw. dass der Ehegattenunterhalt zahlende Vater auch den Kindesunterhalt zahlte. In den westlichen Bundesländern bestand zunächst auch kein Anspruch auf Kinderbetreuung durch den Staat. Der Anspruch auf einen Kindergartenplatz für die Drei- bis Siebenjährigen wurde erst 1992 in das SGB VIII aufgenommen und weit später erst flächendeckend umgesetzt. Der Anspruch auf eine Ganztagsbetreuung kam zudem erst 1996 in das Gesetz.

Allerdings bewirkte der Rechtsanspruch auf einen Kindergartenplatz für die über Dreijährigen eine veränderte Betrachtungsweise im Rahmen des öffentlichen Leistungsrechts. Dies wurde mit der Einführung der sogenannten Hartz IV-Gesetze deutlich, die 2005 das öffentliche Leistungssystem reformierten. Es wurde dabei grundsätzlich unterschieden nach Leistungen für erwerbsfähige Leistungsberechtigte, diese finden sich im SGB II, und für nicht erwerbsfähige Leistungsberechtigte. Diese noch übrig gebliebenen Regelungen der klassischen Sozialhilfe finden sich im SGB XII. Die Erwerbsfähigkeit nach SGB II ( 88 SGB II) ist gesundheitlich definiert und nicht etwa an die familiäre Situation, wie Kinderbetreuung, geknüpft. Die Berücksichtigung der Kinderbetreuung findet sich im SGB II nur im Zusammenhang mit der Zumutbarkeit der Arbeitsaufnahme ( $\mathbb{1 0}$ Abs. 1 Nr. 3 SGB II). Danach kann sich der betreuende Elternteil nicht darauf berufen, dass der Aufnahme einer Erwerbstätigkeit die Kinder- betreuung entgegensteht, wenn das Kind das dritte Lebensjahr bereits vollendet hat und ausreichend Betreuungseinrichtungen zur Verfügung stehen. In der Gesetzesbegründung zum SGB II wird ausdrücklich der Anspruch von Kindern über drei Jahren auf einen Kindergartenganztagsplatz als Grund angeführt, wobei davon ausgegangen wurde, dass der Anspruch flächendeckend umgesetzt sei.

Diese Änderungen im öffentlichen Leistungsrecht waren mit verantwortlich für den heutigen Rechtszustand im zivilrechtlichen Unterhaltsrecht. Eine grundlegende Wandlung erfuhr der sogenannte Betreuungsunterhalt durch die Unterhaltsreform 2008. Der Gesetzgeber ist hier mit dem ausdrücklichen Ziel angetreten, die Chance auf eine Zweit- und vielleicht auch Drittfamilie zu ermöglichen, was sich, ohne dass es ausdrücklich in der Gesetzesbegründung so benannt wird - in erster Linie auf die Männer bezog. Wenn der Unterhaltspflichtige aber eine Chance auf eine Zweit- oder gar Drittfamilie haben soll, dann müssen logischerweise „Altlasten“ beseitigt werden. Dies wurde ebenso konsequent umgesetzt wie die Forderung des Bundesverfassungsgerichts, den Unterhaltsanspruch für die Betreuung der ehelichen Kinder ( $\mathbb{S} 1570$ BGB) und den für die Betreuung nichtehelicher Kinder ( $\mathbb{S} 16151$ BGB) gleichzustellen. Vor der Reform 2008 war der Anspruch aus $\$ 1615$ l BGB grundsätzlich begrenzt auf den dritten Geburtstag des Kindes und ein Titel war entsprechend zu befristen. Mit der Reform 2008 wurde der Betreuungsunterhaltsanspruch weitgehend einheitlich ausgestaltet. Unter ausdrücklicher Bezugnahme auf den Kindergartenplatzanspruch ab dem dritten Lebensjahr wurde er als sicherer Unterhaltsanspruch, sogenannter Basisunterhaltsanspruch, nur bis zur Vollendung des dritten Lebensjahres des Kindes ausgestaltet und darüber hinaus nur noch als Billigkeitsanspruch. Die rechtliche Konsequenz ist, dass die Mutter - unabhängig davon ob sie verheiratet ist bzw. war oder nicht - sich nur noch bis zum dritten Geburtstag des Kindes frei entscheiden kann, ob sie ihr Kind vollumfänglich selbst betreuen will oder nicht. Eine unterhaltsrechtliche Verpflichtung, also eine Obliegenheit zur Aufnahme einer Arbeitstätigkeit, besteht bis zum dritten Geburtstag des Kindes nicht. Arbeitet die Mutter in dieser Zeit, so ist ihr Einkommen überobligatorisch und wird nur teilweise angerechnet. Die völlige Anrechnungsfreiheit hat der Bundesgerichtshof abgelehnt.

Hat das Kind das dritte Lebensjahr vollendet wird Unterhalt wegen Kinderbetreuung nur noch dann geschuldet, wenn die Mutter wegen der Kinderbetreuung nicht arbeiten kann. Dabei ist durch die Rechtsprechung des Bundesgerichtshofs klargestellt, dass ab dem dritten Geburtstag des Kindes kein Wahlrecht mehr besteht zwischen Kinderbetreuung und Arbeitsaufnahme. Vielmehr besteht die grundsätzliche unterhaltsrechtliche Obliegenheit, eine Arbeitstätigkeit aufzunehmen und zwar auch eine Vollzeitarbeitstätigkeit, es sei denn kindbezogene oder elternbezogene Gründe stünden dem entgegen.

Was darunter zu verstehen ist, hat der Bundesgerichtshof in zahlreichen Entscheidungen erläutert. Zur Veranschaulichung wird auf die Definitionen aus den Unterhaltsgrundsätzen des OLG Frankfurt verwiesen. Durch die seit 2008 geltende Ge- 
setzesfassung und die höchstrichterliche Rechtsprechung ist deutlich geworden, dass es für die betreuende Mutter, die die volle Darlegungs- und Beweislast trifft, sehr schwierig ist, über den dritten Geburtstag des Kindes hinaus Betreuungsunterhalt in einem Umfang zu erhalten, der ihren Bedarf deckt. Vielmehr ist mindestens eine Teilzeitbeschäftigung zu erwarten und bei guten Betreuungsmöglichkeiten auch schon bei kleineren Kindern eine Ganztagstätigkeit. Auch ist es der betreuenden Mutter zuzumuten, zu arbeiten, wenn der andere Elternteil verlässlich anbietet, dass er die Betreuung zeitweise übernehmen will um damit die Mutter zu entlasten - was naturgemäß bei hoch zerstrittenen Eltern für viel Zündstoff sorgt. Natürlich bedeutet die Einschränkung des Betreuungsunterhaltes für die geschiedenen oder getrennt lebenden Ehefrauen nicht das Ende des Ehegattenunterhaltsanspruches. Dieser kann immer noch auch aus anderen gesetzliche Vorschriften hervorgehen. Die nicht verheiratete Mutter allerdings bekommt mangels solcher Vorschriften keinen weiteren Unterhalt mehr, wenn der Betreuungsunterhaltsanspruch endet.

Des Weiteren wurden im Zuge der Unterhaltsreform 2008 die Rangfolgen im Unterhaltsrecht geändert. Die Unterhaltsansprüche aller minderjährigen Kinder sowie die Unterhaltsansprüche der den minderjährigen Kindern gleichgestellten privilegierten Volljährigen, also der Kinder zwischen 18 und 21, die unverheiratet sind, bei einem Elternteil leben und sich in allgemeiner Schulausbildung befinden, gehen allen anderen Ansprüchen vor. Im zweiten Unterhaltsrang befinden sich dann die Unterhaltsansprüche wegen Kinderbetreuung und bei langer Ehe. Die restlichen Ehegattenunterhaltsansprüche finden sich erst im dritten Rang. Dies hat zur Folge, dass ein Betreuungsunterhaltsanspruch auch dann scheitern kann, wenn der Unterhaltspflichtige nur so viel Einkommen hat, dass dieses gerade für den Unterhalt aller Kinder aus verschiedenen Verbindungen reicht. Dann bleibt für die betreuende Mutter kein Geld mehr übrig und sie ist auf öffentliche Leistungen angewiesen.

Durch die Einführung des Elterngeldes anstelle des Erziehungsgeldes wurde seit 2007 die Familienförderung verändert. Die Elterngeldleistung knüpft von der Höhe her an das frühere Einkommen des Beziehers an und besteht aus zwei Elementen. Zum einen aus einer Sozialleistung in Höhe von in der Regel 300 Euro, die jeder betreuende Elternteil unabhängig davon bekommt, ob er vorher gearbeitet hat, und dem darüber hinausgehenden Betrag, der vom früheren Einkommen abhängig ist und eine Lohnersatzleistung darstellt. Daraus folgt, dass dieser über 300 Euro hinausgehende Betrag unterhaltsrechtlich als Einkommen einzusetzen ist, was beim Betreuungsunterhalt bedeutet, dass dieser den Unterhaltsanspruch mindert. Im öffentlichen Leistungsrecht ist sogar der Sockelbetrag von 300 Euro auf andere Sozialleistungen (SGB II) anzurechnen, wenn er nicht in dieser Höhe aus Arbeitstätigkeit herrührt. Hier steht der SGB II beziehenden Mutter, die vor der Geburt nicht gearbeitet hat, durch das Elterngeld keinen Cent zusätzlich zur Verfügung.

Und die Zukunft? Auch ohne den Blick in die Glaskugel zu beherrschen, kann man sagen, dass die neuen Regelungen im SGB VIII und im Elterngeldgesetz - gemeint sind der Anspruch auf Kinderbetreuung ab einem Kindesalter von einem Jahr, geregelt in $\$ 24$ SGB VIII, und auf Betreuungsgeld, geregelt in \4a bis $4 \mathrm{~d}$ Bundeselterngeld- und Elternzeitgesetz (BEEG) Auswirkungen auf die Unterhaltsrechtsprechung haben werden. Der Betreuungsunterhalt über den dritten Geburtstag des Kindes wird noch schwieriger zu erreichen sein, wenn das Kind doch schon ab einem Jahr an die Fremdbetreuung gewöhnt werden und die Mutter ihr Leben schon so organisieren konnte, dass sie Kinderbetreuung und Arbeitsaufnahme unter einen Hut bekommt.

Zum Abschluss bleibt festzuhalten, dass die bisher erreichte Gleichstellung ihren Preis hat und zwar auch in unterhaltsrechtlicher Hinsicht. Die gesetzlichen Regelungen sind keineswegs so schlecht, wie sie manchmal dargestellt werden. Es fehlen vielmehr die gesellschaftlichen und vor allem arbeitsmarktbezogenen Rahmenbedingungen, die es den betreuenden Müttern erlauben, Beruf und Kinderbetreuung zu vereinbaren. Hier können wir viel von unseren skandinavischen Nachbarn lernen. Durchgesetzt hat sich inzwischen auch im Westen die Erkenntnis, dass es keineswegs schädlich sein muss für die Entwicklung von Kindern, wenn diese nicht bis zum 18.Lebensjahr ausschließlich von der Mutter betreut werden. Auch entspricht es - hoffentlich - inzwischen dem Selbstverständnis der Frauen, nicht durch eine Ehe oder eine Beziehung in Abhängigkeit zu geraten, sondern für sich selbst sorgen zu können.

Allerdings bleibt ein Punkt, der zeigt, dass gerade bei den Frauen (und natürlich auch Männern in gleicher Situation), die sich bemühen, dem oben genannten Selbstverständnis gerecht zu werden, ein Nachteil entstehen kann, mit dem sie (oder er) nicht wirklich rechnen konnte, weil es nicht den eigenen Unterhaltsanspruch, sondern den des Kindes betrifft. Gemeint ist hier die Regelung des $\mathbb{} 1603$ Abs. 2 S. 3 BGB und die Rechtsprechung dazu. Wenn der unterhaltspflichtige Mann nach Abzug des Kindesunterhaltes nur noch über Geldmittel in Höhe des kleinen Selbstbehaltes von 1.000 Euro verfügt, die betreuende Mutter, die arbeitet und selbst für ihren Unterhalt sorgt, nach fiktivem Abzug des Kindesunterhaltes noch mehr als den großen Selbstbehalt von 1.200 Euro behält, muss dem Vater ebenfalls ein höherer Selbstbehalt als 1.000 Euro zugebilligt werden. Dann muss dieser weniger bis hin zu keinem Kindesunterhalt mehr zahlen, weil er nicht leistungsfähig ist. Dies hat dann zur logischen Folge, dass die Mutter nicht nur die Betreuung des Kindes übernimmt, sondern sich auch am Barunterhalt beteiligen oder gar allein dafür aufkommen muss.

Hier wird ganz sicher die (der) Tüchtige bestraft! 